\title{
Healthcare providers' perspectives on parental health literacy and child health outcomes among Southeast Asian American immigrants and refugees
}

\author{
Belle P. Khuu ${ }^{\mathrm{a}, *}$, Hee Y. Lee ${ }^{\mathrm{a}}$, Anne Q. Zhou ${ }^{\mathrm{b}}$, Jihee Shin ${ }^{\mathrm{a}}$, Richard M. Lee ${ }^{\mathrm{b}}$ \\ a School of Social Work, University of Minnesota, St. Paul, MN, USA \\ b Department of Psychology, University of Minnesota, Minneapolis, MN, USA
}

\section{A R T I C L E I N F O}

\section{Article history:}

Received 10 February 2016

Received in revised form 6 June 2016

Accepted 7 June 2016

Available online 8 June 2016

\section{Keywords:}

Health literacy

Parental health literacy

Healthcare disparities

Children health

Immigrants

Refugees

Asian Americans

Health outcomes

\begin{abstract}
A B S T R A C T
Low health literacy has emerged as an important area of research because of its close link with health disparities. In this study, we used a qualitative approach to investigate healthcare providers' perspectives on the health literacy of immigrant and refugee parents and its association with children's health. Sixteen health and mental health professionals serving immigrant and refugee parents and children in various clinical settings were recruited through a purposive sampling method and interviewed. Six broad themes were identified: (1) multi-dimensional components of parental health literacy; (2) parent characteristics and native country experiences; (3) host systems and their interactions impact on parental health literacy; (4) diverse aspects of help-seeking; (5) culture-based parental help-seeking; and (6) child health outcomes. Within these larger themes, the complexity of parental health literacy and its various effects on children's health outcomes among immigrant and refugee parents were evident. Future research includes more population-based quantitative studies of parental health literacy and culturally relevant clinical approaches among immigrant and refugee parents.
\end{abstract}

Published by Elsevier Ltd.

\section{Introduction}

Health literacy refers to the degree to which individuals have the capacity to obtain, process, and understand basic health information and services needed to make appropriate health decisions (Ratzan \& Parker, 2000 p. vi). It is often conceptualized as a core set of knowledge and skills that are essential to functioning in a healthcare system (Baker, 2006). Although more attention has been paid to "physical," rather than "mental," health in the empirical literature, our conceptualization of health literacy encompasses both domains and aims for a more holistic perspective and integration of health (Jorm, 2012; Sørensen et al., 2012). One major public health issue associated with health literacy is the prevalence of low health literacy in the United States; approximately 80 million, or nearly half of the adults have limited or low health literacy (Nielsen-Bohlman, Panzer, \& Kindig, 2004). Adults with low health literacy are less likely to seek preventive care services, more likely to use emergency department visits, and require more resource intensive services, such as hospitalizations (Berkman, Sheridan,

\footnotetext{
* Corresponding author at: University of Minnesota, School of Social Work, 105 Peters Hall, 1404 Gortner Ave., Saint Paul, MN 55108, USA.

E-mail address: bkhuu@umn.edu (B.P. Khuu).
}

Donahue, Halpern, \& Crotty, 2011). In addition, low or limited health literacy has been linked with negative physical and mental health outcomes. Patients with low health literacy are more likely to report symptoms of depression (Coffman \& Norton, 2010), less likely to take appropriate medications (Kripalani et al., 2006), and less likely to receive immunization against influenza (Bennett, Chen, Soroui, \& White, 2009). However, current research on health literacy has focused mainly on adult health outcomes. There is limited research on the relations between parental health literacy and children's physical and mental health outcomes.

Parents serve as a major determinant of their children's developmental outcomes (Glascoe \& Dworkin, 1995). Yet, a nationally representative survey of 6100 parents found that nearly one out of three parents has low health literacy (Yin et al., 2009). This low health literacy can potentially have a deleterious effect on children's physical and mental health trajectories, as parents with low health literacy are more likely to have children without insurance and have greater difficulty understanding medication and nutrition labels. Given the integral role parents play in child development, it is important to gain a better understanding of how parental health literacy affects child outcomes (Sanders, Shaw, Guez, Baur, \& Rudd, 2009). However, findings from the current literature on the impact of parental health literacy on their children's physical and mental health outcomes are mixed and vary based on the population studied (DeWalt \& Hink, 2009). 


\subsection{Parental health literacy within immigrant and refugee families}

Recent studies have indicated that some parent populations, such as immigrant and refugee parents, are particularly at risk for low health literacy (Kreps \& Sparks, 2008). In a study of immigrant caregiver-child dyads, one in five caregivers had low health literacy (Sanders, Thompson, \& Wilkinson, 2007). Furthermore, being foreign-born, having limited English proficiency, and lacking a high school education have been found to be the strongest predictors of low health literacy among these caregivers. Parents with limited English proficiency are more likely to use urgent care services and child health services in general (Sanders et al., 2007). Additionally, children who are foreign-born or naturalized citizens born to foreign-born or immigrant parents are less likely to have visited a mental health provider in the preceding year (Huang, Stella, \& Ledsky, 2006). Compared to children of parents who are citizens, children of non-citizens are less likely to have access to medical and dental care and thus have delayed care (Yun, FuentesAfflick, Curry, Krumholz, \& Desai, 2013). Although the research in this area is limited, the findings suggest that it is crucial to examine more thoroughly parental health literacy among immigrant and refugee families.

\subsubsection{Health providers' perspectives on parental health literacy}

Even though health literacy is often conceptualized as the patient's or the individual parent's capacity for health information, healthcare providers play a crucial role in assessing and intervening in regards to parental health literacy (Hironaka \& Paasche-Orlow, 2008). Healthcare providers frequently interact and communicate with patients who have a diverse range of health literacy levels. Through these interactions, providers acquire a unique vantage point that needs to be further explored (Dennis et al., 2012). In a national survey of pediatricians, for instance, $81 \%$ of pediatricians were able to recall a clinical encounter in the past 12 months in which a parent displayed signs of insufficient understanding of health information provided. Additionally, $44 \%$ of these physicians were fully aware of an error in communication pertaining to patient care in the previous 12 months (Turner et al., 2009). Therefore, issues regarding low or limited health literacy are occurring frequently during clinical encounters, and these issues can greatly impact both the patient-provider relationship and their clinical interaction (Ali, 2012; Rosenthal et al., 2007).

When healthcare providers deem their patients to have low health literacy, it can impact their subsequent treatment of patients. Harrington, Haven, Bailey, and Gerald (2013) found that healthcare providers' perception of the parents' health literacy influenced their asthma treatment recommendations and instruction provided to the patients. Specifically, patients' verbal responses and their past behavior regarding asthma management were taken into consideration by healthcare providers. These health literacy assessments were also influenced by patient-specific characteristics. Patients identified as White were more likely to be designated as having adequate health literacy compared to patients identified as Black. This finding suggests that the patients' racial and ethnic makeup plays a role in the healthcare providers' assessment of patients' health literacy and subsequent intervention approaches. Thus, a closer examination into healthcare providers' perspectives is warranted to understand the impact of parental health literacy on child outcomes.

\subsection{Conceptual framework}

To guide our investigation of healthcare providers' perspectives, we developed a conceptual framework derived from the extant physical and mental health literacy literature (Fig. 1). The conceptual framework highlights the possible factors impacting parental health literacy, including cultural influences, parents' prior interaction with their native country's existing health systems, individual characteristics, and host systems such as schools and healthcare. Parental health literacy was conceptualized as a multi-dimensional construct that encompasses both physical and mental health and includes the domains of cognition, behaviors, and attitude. In turn, parental health literacy was conceptualized as a determinant of health behaviors, including parental help seeking and whom the parents seek help from. In this conceptual model, parents are viewed as active agents who gather recommendations from the sources of help but ultimately must make the decisions about whether to take further action. Parental health literacy, therefore, remains the central bridge between parents and their social and health environment, acting as a factor that helps parents to make health decisions and influences their behaviors. These

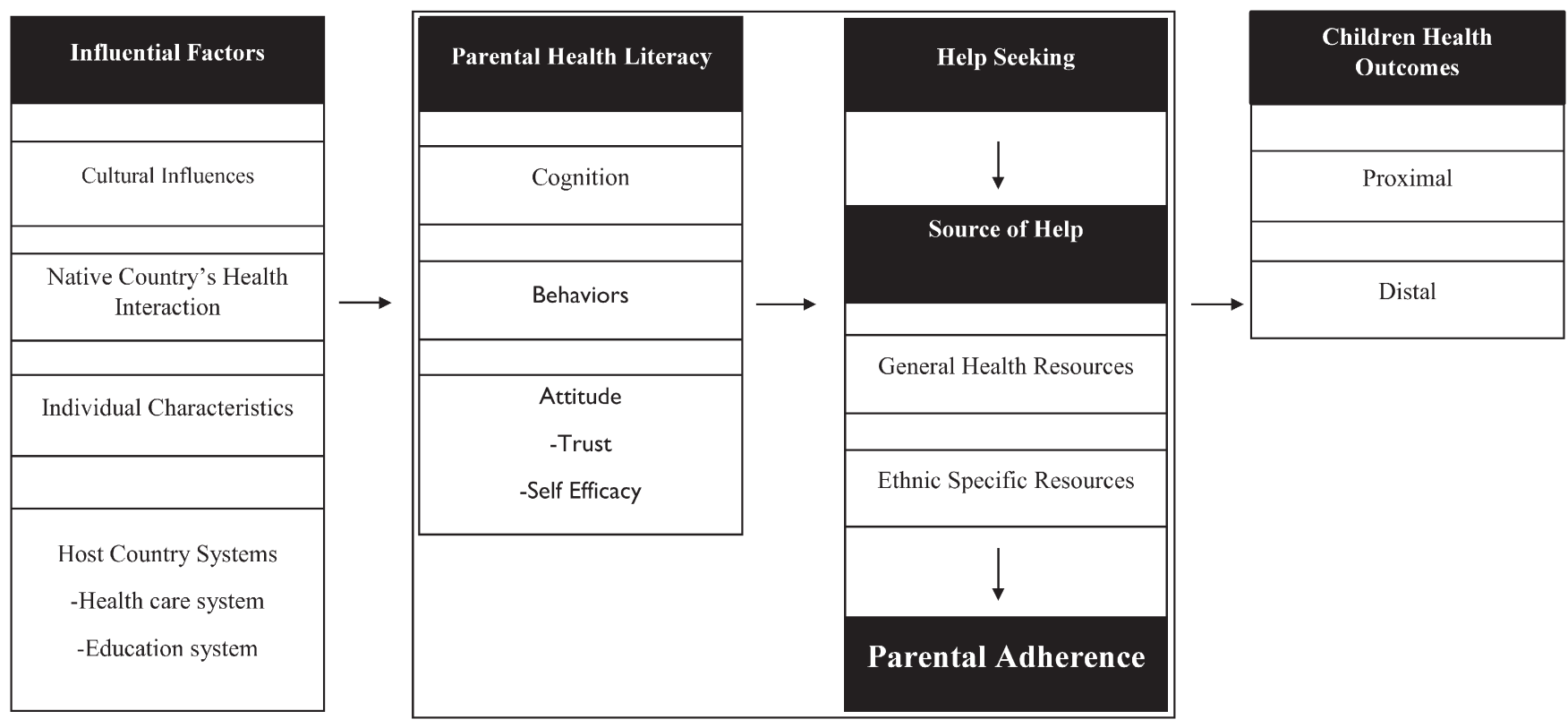

Fig. 1. Immigrant and refugee's parental health literacy conceptual model. 
decisions, attitudes, and behaviors subsequently have an impact on their children's health outcomes. These outcomes can be both proximal and distal, and include desirable and undesirable outcomes (e.g., lack of access to healthcare leading to escalation or prolonged illness) among children.

\subsection{Current study}

Although health literacy is considered a critical factor in determining health disparities and child development outcomes, there is very limited research on immigrant and refugee parents, particularly within the Asian American community and its diverse ethnic sub-groups (Han, Kim, Kim, \& Kim, 2011; Abe-Kim et al., 2007). Given that Asian Americans make up a significant portion of the immigrant population in the US (Pew Research Center, 2012), a deeper understanding of these parents' health literacy and its impact on children's health is necessary and timely. We employed a qualitative approach to explore healthcare provider perspectives on the impact of parental health literacy on children. We specifically sought out the views of healthcare providers who mainly work directly with immigrants and refugees due to their clinical experience in caring for patients with diverse health literacy levels. This qualitative approach enables a more in-depth information gathering on healthcare provider perspectives of parental health literacy and its impact on children's health outcomes. This study addressed gaps in the literature by investigating (1) the current level of health literacy among immigrant and refugee parents as perceived by healthcare providers, (2) its impact on parental help-seeking behaviors, and (3) and its effects on children's health outcome.

\section{Methods}

\subsection{Study procedures and data collection}

This study employed a qualitative research method approach using individual in-depth interviews which was approved by the University of Minnesota's Institutional Review Board. Participants were recruited through a purposive sampling method where potential participants were recruited based of their professional affiliations and clinical reputation within certain clinics, agencies, and hospitals serving mostly immigrants and refugee populations unique to this Midwestern state. Participants who were eligible included physical and mental healthcare providers who were currently working or had worked with a substantial number of Southeast Asian and African immigrants and refugees for a majority of their clinical career. These providers had self-identified as providing or having provided physical and mental healthcare services to immigrants and refugees in either clinical training or practice settings. Participants were sent study questions prior to being agreed to be interviewed and were offered the choice to be interviewed at the second author's office on the university campus, the participant's own office, or another private and quiet location of their choice. In total, there was 16 study participants $(\mathrm{N}=16)$. Eleven of the participants chose to be interviewed at the second author's office while the rest opted for their own office. The first and second authors conducted all the interviews.

The interviews used a semi-structured approach, following a set of research questions with prompters (Appendix A). Interviewers were allowed to follow-up with statements participants made, with prompts and clarifying questions, at their discretion. The interviews lasted approximately 90-120 min and were conducted in English. Interviews were conducted until the point of data saturation, which was determined among the interviewers through weekly meetings and continual reflection of the incoming data. Data were collected from May 2012 to November 2012. All interviews collected were transcribed by two masters-level students and one undergraduate-level student.

\subsection{Data analysis}

The data were analyzed using the thematic approach analysis developed by Braun and Clarke (2006). Two coders, the first and fourth authors, independently analyzed the entire data set. The first author is a doctoral student, immigrant, and bilingual in English and Vietnamese. The fourth author is a masters-level international student who is bilingual in English and Korean. The process began with both coders reading every transcript multiple times and identifying emerging trends and repeated ideas. Main meanings through key phrases, sentences, and ideas were also highlighted to capture thematic components surrounding parental health literacy during the independent open coding phase. Common aspects of each idea and relational statements were then categorized and initial codes were developed. The identified ideas were then grouped using the initial codes. Themes and subthemes were identified to further subcategorize the data afterward. Throughout the process, the themes were identified, reviewed, labelled, and tested through comparison of fit between the ideas and broad themes. The coders met to discuss coding and rationale, addressed discrepancies, and recoded when necessary. Major discrepancies that were deemed as irreconcilable were settled by the second author who is bilingual in English and Korean. Consensus coding processes were later employed to assess the agreement of thematic interpretation and development. Furthermore, underlying concepts and themes were continually discussed at team meetings.

\section{Results}

\subsection{Sociodemographic characteristics of the sample}

There were a total of 16 participants with ages ranging from 25 to more than 65 years, with a mode of 45 to 55 years of age. Among participants, there were five physicians-of whom two were psychiatrists-three social workers, two psychologists, two mental health clinical supervisors, one social work assistant professor with clinical background, one mental health case manager, one community health worker, and one nurse. Five of the participants worked mainly with adult populations, while the rest worked with both adults and children. Of the 16 participants, four participants reported being born outside of the United States. In regards to ethnic racial status, seven participants identified as Asian Americans and nine as Non-Hispanic Whites $(n=9)$. Asian American health providers included Hmong $(n=3)$, Vietnamese $(n=2)$, Chinese $(n=1)$, and Korean Americans $(n=1)$. The reported mean number of years of experience was 19 years $(S D=13)$ with a range of four to more than 37 years. The immigrant and refugee populations that these healthcare providers served include Hmong, Vietnamese, Laotian, African (Somali and Ethiopian), Korean, Lebanese, Bhutanese, Latino, and Cambodian Americans. The demographic information of all participants is available in Table 1.

\subsection{Themes developed from the interviews with healthcare professionals}

Data analysis revealed six overarching themes that emerged during the interviews with the participants: (1) multi-dimensional components of parental health literacy; (2) parent characteristics and native country experiences; (3) host systems and their interactions impact on parental health literacy; (4) diverse aspects of help-seeking; (5) culture-based parental help-seeking; and (6) child health outcomes. The complexity of parental health literacy and its various effects on children's health outcomes were apparent throughout all these themes.

\subsubsection{Multi-dimensional components of parental health literacy}

The majority of the healthcare providers suggested that many of their patients demonstrated low levels of health literacy. Several providers specifically mentioned lack of awareness in the parents as a critical influence in the providers' perception of health literacy. A White 
Table 1

Participants' demographic information.

\begin{tabular}{|c|c|c|c|c|c|}
\hline Participant & Sex & Occupation & Ethnicity & Age range & Years of experience \\
\hline 1 & Female & Psychologist & White & $45-55$ & 28 \\
\hline 2 & Female & Social worker & White & $55-65$ & 12 \\
\hline 3 & Female & Community health worker & Vietnamese American & $45-55$ & 14 \\
\hline 4 & Male & Social worker & White & $45-55$ & 16 \\
\hline 5 & Female & Sw ${ }^{1}$ assistant professor & Hmong American & $35-45$ & 5 \\
\hline 6 & Male & Physician & Hmong American & -3 & -3 \\
\hline 7 & Female & Physician & Chinese American & $25-35$ & 4 \\
\hline 8 & Male & $\mathrm{MH}^{2}$ case manager & Vietnamese American & $25-35$ & 8 \\
\hline 9 & Male & $\mathrm{MH}^{2}$ clinical supervisor & White & $55-65$ & 36 \\
\hline 10 & Female & Psychologist & White & $45-55$ & 24 \\
\hline 11 & Male & $\mathrm{MH}^{2}$ clinical supervisor & White & $55-65$ & 40 \\
\hline 12 & Male & Physician & Korean American & $45-55$ & 19 \\
\hline 13 & Male & Psychiatrist & White & $65+$ & 37 \\
\hline 14 & Female & Social worker & Hmong American & $25-35$ & 3 \\
\hline 15 & Male & Psychiatrist & White & $65+$ & 37 \\
\hline 16 & Female & Nurse & White & $25-35$ & 4 \\
\hline
\end{tabular}

${ }^{1}$ Social work. ${ }^{2}$ Mental health. ${ }^{3}$ Did not provide information and was excluded from subsequent analysis.

nurse with four years of clinical experience said, "If they're not aware there's being a problem. That is just the whole locus of control. It's a hard system and without that health literacy, it's a very different world than where they're from; I can't say that enough." This quote illustrates the conception that immigrant and refugee parents were deemed by some health providers as not able to recognize their inability to understand basic health information. Their lack of understanding ranged from not knowing the etiology of their child's ailment, the differentiation of the various types of health conditions such as chronic versus acute onset, or the phases of various illnesses such as initial to terminal. Additionally, the providers conveyed that sometimes their patients did not know the purpose of their visit or of the different healthcare settings such as acute clinics, emergency rooms, primary care clinics, and specialty clinics. The same parents were also perceived not to have knowledge regarding health provider specialties and common procedures during a health visit, follow-up care, and treatment plans.

However, others healthcare providers challenged the notion of the complete lack of awareness of health and disease among immigrant and refugee parents. These providers conceptualized their patients not as being unaware of certain health knowledge (e.g. knowledge of health conditions) or having low health literacy, but rather conveyed that there may be a difference in conceptualizations of illness among different cultural groups. A Hmong American physician provided an example of how illness could be conceptualized differently within Eastern and Western cultures: "For instance, I take my five year old, we went to-, it's nice and but chilly and windy and we went to soccer tournament, we came back and may caught cold right - they would say that she got sick because wind caused it." Another provider, a Hmong American social worker with 5 years of experience, echoed this idea of a categorically different conceptualization of health literacy. Furthermore, the social worker further asserted that health literacy, for some patients, is actually high instead of low or marginal by stating, "I think they have health literacy within their own culture, so they can communicate that very well, but they don't have health literacy in what mainstream or Western systems...So they're unable to communicate that very well - so those are two things going on there."

In other words, the healthcare provider perceptions of low health literacy in the immigrant and refugee populations may not mean a lack of awareness and knowledge but may mean instead that there are differences in conceptualizations of health and illness between the provider and the patient. As such, the patient is not truly health illiterate and may just have low literacy regarding Western connotations of health and health services. The refugee and immigrant parents were perceived by some providers to process and communicate medical information differently from the Western medical community. The core differences in conceptualization of health literacy demonstrated the need for a health literacy concept for the immigrant and refugee population that includes the degree of congruence or discordance between the parents' and healthcare providers' conceptualizations of health and health literacy. Doing so will help foster and sustain the therapeutic relationship between patients and their providers.

Other domains of health literacy identified were attitudes and perceptions toward the healthcare system, such as the expectations for care and developing trust. For example, a White psychologist with 28 years of experience said, "It's not just information that people need about a health condition, but an idea about how this [Western health system] could be helpful." This quote conveys the importance of understanding not only health knowledge but also the parents' underlying attitudes toward the Western healthcare system. In addition, one clinical White supervisor with 36 years of experience mentioned, "If you [patient] can't call someone and be confident they're gonna pick it up in your language - that's gonna impact that kind of behavior." Thus, issues such as language and cultural barriers remain important aspects impacting parents' attitude toward, and trust in, the healthcare system and providers.

Providers identified the health communication skills of parents as another crucial component of health literacy where effective health communication was viewed as directly related to health service seeking. In addition, parents' health communication ability was viewed as directly impacting the quality of health interaction with providers. For example, a Vietnamese American community healthcare worker with 14 years of experience said:

Health literacy, if a person has more knowledge about that, that will help them presumably communicate with a healthcare professional more effectively if they have a question about health, then it's easier for them to communicate it. If a person has knowledge about health literacy, then they're more understandable about the material, so they might have better questions when communicating with health providers.

Within the skill domain of health literacy, healthcare providers viewed not only knowledge but also the openness and willingness of the patient as an integral component of health literacy. The same provider added:

Also, if they have knowledge of the healthcare system, then it's easier for them to access what the healthcare system told you, that the more understandable about healthcare asset and healthcare literacy, the more susceptible at seeking for help and more accepted into the healthcare system and more questions to healthcare provider and healthcare professionals.

Thus, health literacy appeared to include not only health information that parents processed and obtained, but also the attitude and skills that 
parents develop that help them navigate and interact with the healthcare system. These findings suggest that health literacy has a complex and dynamic nature, and that it is interrelated with constructs such as help-seeking and service utilization.

\subsubsection{Parent characteristics and native country experiences}

Another common theme was the various ways in which exposure and experience with the healthcare system in the parents' native country made an impact on parental health literacy. Nativity status and country origins are two relevant factors. It matters whether parents came from a country that had a Western model or system of healthcare that emphasized and practiced preventive care versus indigenous healthcare services that were used only for emergencies. A White clinical supervisor with over 36 years of clinical experience said, "If the parents grow up without immunization in the old country, and then they grow up healthy without major disease, they may think their kids don't need to do it." Thus, prior to immigrating to the United States, the parent's health literacy is already shaped by their experiences with the healthcare system in their country of origin. A White psychologist with 28 years of experience said:

We also have a number of immigrant communities in this area that $\mathrm{I}^{\prime}$ $\mathrm{m}$ familiar with who don't have experience with Western healthcare systems. Their primary experience with health conditions is for acute conditions where they have to travel quite a long time to see a healthcare provider, and it's for something that could be life threatening or a very serious condition.

Along with exposure to the Western model of healthcare systems, broader cultural and social influences can impact the parents' health literacy levels. Some of these influences included general beliefs regarding children and their developmental trajectory and needs, beliefs about health and illnesses as well as ways to remedy those illnesses, along with world view regarding Western medicine, including the roles that health experts have to impact those illnesses. A White clinical supervisor with over 36 years of clinical experience said,

I think about the communities that don't get to us-and those are communities like the Hmong community who simply have a different way of solving problems-that they're experiencing autism for their children just as much as other communities are, but their response to the problem, and how they help that condition, is very different. And so, the invitation to various immigrants really depends on their starting point.

In addition, the participants cited individual characteristics of the immigrant and refugee parents as a major influence of their level of parental literacy. These individual characteristics included illiteracy and lack of English literacy, education, income, and employment level among the parents, with the majority of the participants relaying that the families they served were mostly at or below poverty level. A White clinical supervisor with over 40 years of experience stated that he believed poverty was an important factor because "they would just pass onto their children to have hesitance for them to get... for instance... immunization or even go to the doctors." Thus, the impact of economic deprivation was passed down from parents to children by influencing the parents' attitude and understanding toward health service and preventative service use, which in turn affected the health decisions and actions parents made regarding their children's health. Other providers specifically mentioned insurance status as an important factor of whether parents accessed the healthcare system, enabling parents to act on behalf of their children.

Providers also suggested that immigration status and acculturation served as relevant parental characteristics contributing to parental health literacy. They indicated that their patients' documentation status affected their patients' legal access to the healthcare and health insurance, as well as their trust in the healthcare system. Specifically, the providers mentioned that undocumented immigrants were especially difficult to reach because of their fear of deportation and distrust in the healthcare system. However, there was mixed consensus whether these issues were similarly present in refugee populations as the immigrant populations. Some speculated that the legal designation of immigrants secondary to their voluntary migration status, especially those sponsored by other family members or organizations, were worse off due to policies that prohibit them from automatically receiving health benefits. In contrast, those who arrived in the United States with the legal status as refugees were able to receive more benefits and services, which included access to healthcare. Other participants saw these differences as merely legal or official categories, and connoted that there was little differentiation between the groups in regards to parental health literacy. In addition to immigration status, differences in acculturation level were also mentioned as influencing the varying levels of familiarity with health clinics and key players within the health systems, as well as health information. Parents who were more acculturated were believed to have had higher health literacy because of their greater exposure to the mainstream society.

Furthermore, many of the providers, especially those working in the mental health field, discussed at length how the parents' trauma could serve as a major impediment to their health literacy. Several of the providers reported that their patients emigrated from areas of major political and social unrest that played a major role in their patients' mental health and psychological distress. The psychologist with 28 years of experience said:

So often I find that parents who are depressed have less capacity to care for their children who may also be experiencing depression or anxiety, and so their ability to be attuned to their child's needs, to even take in information that I'm trying to share with them about their child's needs, and then to act on that, those are several hurdles that they have to overcome in order to be able to do that.

The traumatic experiences encountered in their native country and disposition of the parents were perceived as influencing their ability to obtain and use health information to make the needed health decisions for their children.

\subsubsection{Host systems and their interactions impact on parental health literacy}

For many parents, schools serve as a major influence in providing health information and access to health services. A common theme in the interviews was the interaction between the parents and schools. Though non-immigrant and refugee parents also have close relationships with their children's schools, for immigrant and refugee parents the boundary between the school and healthcare system is often blurred and complicated. A White psychologist with 28 years of experience stated:

I would say, in all the years I've worked with children, at least half the time they've come through the school system. And so, families will come because a teacher raises concern, or because their child is starting school but the people who evaluated them to get them started said that they're not learning on track-those are the situations that end up then coming to parents' attention. And it's not as though parents don't understand that there's a need that their child has, or there's a problem-so they will say, well, "of all my children, this child was the one who always had difficulty learning," or "I always knew that they were going to have some problems adjusting to school."

While some parents are cognizant of their children's problems, there are other health issues which they may not understand or be aware of, especially when it comes to preventive care issues such as vaccinations and wellness checks. The lack of full understanding of the purpose of and appreciation for mandated health services by the parents could influence the health interaction and outcome between families and their providers. A Hmong American physician remarked: 
Some of the patients we have, or parents, they bring their children to get vaccinated only because they understand that if they don't get vaccinated, they don't get to go to school. I understand that part is important, but what is more important is that parents understand that vaccinations are to prevent disease, not just so they can go to school. So when you have a parent that doesn't have that understanding, you have children who don't get vaccinated according to the right schedule, or never. And then they go to school and are sent from the school to get vaccinated, and then so if you don't understand what is needed to be done at a routine physical, or if you don't know why you need a routine physical you may go to see your doctor, and you don't want to do anything.

Additionally, healthcare providers recognized the impact institutions could have on parental health behaviors by indicating the importance of institutions in facilitating engagement between the health community and parents. Some providers commented on the importance of engaging not only with the parents but also with schools in order to improve the assessment of health issues among teachers. Others discussed the issue of connecting with other non-school institutions, such as ethnic-specific community organizations as well as "frontline" workers. One White psychologist with 28 years of experience stated:

I think, for many years, my thought was that psychologists need to do a better job of being out there in the community and being seen as a first step for mental health problems-but I think that's not really understanding our own culture. I don't think psychologists-at least in the foreseeable future-are going to be out there in the way that doctors are. It's just not going to happen. So I think really what we have to do is become better partners to those people who are the gatekeepers, who are the first steps for families, because that's where the problems are being first communicated.

Thus, healthcare providers suggested that improving parental health literacy would also require improving the inner workings of the various systems such as schools and clinics as much as improving the relations between these two systems. Subsequently, the degree to which these systems are able to interact with the parents and jointly among themselves to foster purposeful collaboration among all the systems is vital to parental health literacy. Moreover, the ability of health and school systems to better recognize their respective strengths and limitations regarding immigrant parents' health could be vital in aiding parents to become better health advocates for their children.

\subsubsection{Diverse aspects of parental help-seeking}

Help-seeking, within the context of health literacy, was revealed to be an important factor impacting child health outcomes. In the interviews, some providers talked about help-seeking not merely as parents seeking help from other providers but also as the different ways in which they sought help. Several providers stipulated that immigrant and refugee parents may not seek help for a variety of reasons. A Hmong American social worker with three years of experience posited, "The parents may not understand that, that may impact their child's mental health because that is what they are used to. So that is normal too, so they may say, I experienced that too; maybe my child doesn't need help." However, another White clinical supervisor added:

When it comes to trauma-related mental health, they are really motivated to ignore because sometimes they themselves are suffering and they deny impact of trauma on their children. When they have spent whole life, you know - war time situation trying to make sure their child doesn't die.

Even though it is only speculation as to whether or not knowledge is a critical aspect toward help-seeking, there may be other psycho-sociological aspects that influence this critical health behavior with regard to formal sectors of health services, such as previous experience with conflicts and trauma.

Another issue that informs help-seeking is the level of understanding and knowledge of the illness. The community health worker with 14 years of experience said:

So, I think that if a family member knows that their child needs help, that they'll do whatever it takes. Because, most of the time, if you ask a refugee why they've come here to our country, it's for their children. It's so their children can get an education, they can have a better opportunity at life, and so they want their children to be healthy and-you know-have a good life.

Although the sentiment of hope for a better opportunity and life for their children is present in many parents, it is especially tangible for immigrant and refugee parents as their countries of origin were often filled with conflict and poverty. This sentiment of wanting the best for their children can trickle down to health beliefs and behaviors, including help-seeking. However, the parents' desire to improve their children's health outcomes is complicated by the fear of legal or social consequences. A Vietnamese American counselor discussed this complication:

Due to the fear - first of all I don't have health insurance - we don't have health insurance and they going to lock my daughter up and they are going to... what if they tell the community? Information get out... we will - my other daughter, will she get married? Due to all of the fear they waited for two years before they come to us to seek help and the stage it was pretty severe so I had to transfer to ACT [Assertive Community Treatment Team], a for help.

As the healthcare system can be challenging even for many mainstream Americans, it is likely a more difficult barrier to overcome for immigrant and refugee parents facing health concerns for their children. A Hmong American primary care provider stated that "One of the things I do notice is that with mental health, some of our patients don't really know how to access mental health resources. I think there is there may be a stigma about getting treatment for mental health as well." Even for the parents that do manage to eventually seek help, the process is also further complicated by other knowledge needed to navigate the health system, such as actual services available, knowing their rights as patients, their ability to articulate and communicate with healthcare providers, and their prior experience with healthcare providers. To these points, A Hmong American social worker said, "I think to just navigate where people need to go is hard, and what services are there, and what rights they have in terms of being a part of their treatment."

\subsubsection{Cultural sources of help-seeking}

When a child is recognized or assessed as having a health or mental health problem, immigrants and refugee parents seek help and services from numerous sources. But instead of seeking help from formal mental health services, they are more likely to go to families, friends, respected individuals in the community, spiritual healers, religious leaders, and other ethnic-specific resources, such as ethnic-specific community centers and organizations. These sources of help sometimes take the place of formal health services or serve as the initial source of care for many of these parents who are faced with mental health concerns rather than services specializing in mental health, such as counselors or psychiatrists. One White psychiatrist with over 37 years of experience remarked, "There is a tendency use to folk remedy whether it's herbal stuff or prayer stuff or Shaman or Imam. So there is delaying in getting into health providers." Another White psychiatrist also with 37 years of experience also stated, "This general, let's say distrust or lack of like familiarity with medical practice, doctors... you don't go there until you're dying."

For others within the community, further factors influence whether and where parents are more likely to seek services. The White clinical supervisor stated: 
And then I think that a lot of parents just treat their own way. We have some parents who are fairly recent refugees that you know also it depends on what kind of environment they came out of. Some came out refugee camps where there was fairly good healthcare, some came from cities where there was access and so they've learned that doctor is good and medication helps. Other people come from really rural areas where you know the closest medical clinic was hundred miles away so they kind of learned to deal with own stuff.

Additionally, many of the providers acknowledge that there are differences in help-seeking between the different Asian American immigrant and refugee ethnic subgroups. One Hmong social worker with five years of experience stated:

And that's why there are lower levels of service utilization among the Hmong, in terms of mental health services, you know, because they just don't see that connection. I mean, I think it's easier for them to go to a physical, medical primary care doctor and talk about mental health. Because for them, mental health means "things are difficult," or "I'm feeling tired," or "I'm feeling ill," "I don't have a lot of energy"-that's mental health. And so for them, that translates into the primary care services, you know.

Another common theme was that many parents demonstrated a preference for seeking help from more general sources of healthcare such as primary care clinics and emergency departments. Many parents also use non-medical sources of care, such school, which for many parents is often the first source of help. A White psychologist with 24 years of experience noted:

You know again that depends on how savvy they are of the western system because some refugees come to thinking that school should solve all of their child's problems and it's up to the teacher and they defer to what just the teacher decides is best for the child. Some parents would be afraid of system because they were afraid of system at their home country. And they won't want to draw attention to themselves especially they are not citizens. If they just have refugee status, they worry that being dependent or getting too much welfare getting attention might get them deported or affected their ability to pay their rent or stay in the country. They have a lot of misgivings about being involved in Western systems of care anyway.

Thus, to understand immigrant and refugee parents' sources of service-seeking, it is important to take into consideration the ethnic affiliation of the parents, type of illness, prior exposure and experience with Western healthcare system, as well as their view of the problem. These factors, in addition to ethnic-specific or more general resources, impact where parents prefer to seek help.

\subsubsection{Parental health literacy and its impact on child health outcomes}

Healthcare providers viewed parental health literacy as critical to child health outcomes. A community healthcare worker with 14 years of experience summed up the relationship between parental health literacy and child health outcomes by saying, "The more they understand, the more they can help their children, and then the children will be healthier and have better results." The participants discussed the impact of parental health literacy in terms of either desirable or undesirable health behaviors. For desirable behaviors, many providers mentioned the active engagement of parents with the healthcare system. A Korean American physician stated, "Certainly I think that those that are more engaged in our healthcare system and understanding healthcare system and our beliefs in health and wellness are more like to take their children in, so I mean that would be the most sure thing I could really say." In turn, these desirable health behaviors result in better child health outcomes, including health problem alleviation, reduction of symptoms, healthier status, and, for some children, a recommencing of their normal lives prior to their illness, including work and productivity. To this point, a Vietnamese American counselor described a clinical case he encountered:

Because they are more open to that once they come via emergency, we came to educate parents what is mental health and everything that able to get this support. Once they know that we're not taking her away, they are more receptive to medication. I think for her, at last I know, she went back to the work force her symptoms are really reduced - the lack of parents understanding the health issues barred the daughter from seeking help in the first place.

Similarly without or with low health literacy, parental health behaviors and actions could lead to undesirable consequences for their children's health. Many providers discussed cases where parents did not seek services for their children, did not engage in care, were unable to fully appreciate the medical consequences of poor health behaviors, delayed help-seeking, lacked follow-up or did not follow the precise medical protocol, were incompliant with treatment, or were uncooperative with the health providers. A Hmong American physician summed up these issues by stating:

For example, we have a child, a small child, that is failing to thrive, and they are not growing, and so with failure to thrive it can cause - it can have negative impact on their mental development, cognitive development, as well as physical development. But the parent and their kids, for whatever reason - and we tried to explain that the child is not growing and needs to go to a feeding clinic and needs to be fed you know through a G-tube, understandably this is a very complicated situation for the parents, um... and I think there is a health literacy, is low health literacy, is one of the reasons that they are incompliant to treatment, but there may be other cultural barriers too, that's why they aren't compliant. And when children are involved and parents are non-compliant and that child patient is eventually going to have a poor health outcome from their condition, then this is when social services get involved and child protection gets involved, and it creates a situation that no one is happy about, not the patient, not the parent, not the providers.

The case the provider mentioned, as well as the other examples provided, illustrates the negative health outcomes for children with parents of low health literacy, such as unmet medical needs worsening the disease, children being frequently sick, increases in other risk factors, or more resource-intensive care being required. This was also true for mental health needs, which were often unmet. As stated by a social worker with 5 years of experience, "I think for the kids, mental health is probably out of the picture most times. They go in for the physical checkups because you are required for children, which are a good thing." Thus, the difference in outcomes was perceived by providers to be tied to the type of health concern and mechanisms, existing or absent, available to ensure the well-being of children.

\section{Discussion}

The purpose of this study was to examine parental health literacy and its impact on their child's health outcomes from the healthcare providers' perspectives. The findings indicated that, when healthcare providers ascribed to Western conceptualizations of physical and mental health literacy, parents were more likely to be deemed by providers as having low health literacy. Providers deemed parents as lacking awareness of basic health information, ranging from disease etiology to treatment to the parental role in child health maintenance. Thus, the present study highlighted the need to broaden the conceptualization of health literacy beyond the sphere of just knowledge and understanding, to attitudes and skills regarding health behavior. This expanded idea of health literacy, especially for the immigrant and refugee population, can be built on the existing understanding of health literacy (Tsai \& 
Lee, 2015). From the providers' perspective, health literacy encompasses the understanding, expectation, and appreciation for the health system in general and for specific agents within the system. These expectations and appreciation add to "the cognitive and social skills which determine the motivation and ability of individuals to gain access to, understand and use information in ways which promote and maintain good health" (World Health Organization, 1998). For many individuals, having access to and understanding health and mental health information may be a sufficient indicator of health literacy. However, for immigrants and refugees, the concept of health literacy must be extended to include attitudes regarding health and the healthcare system, along with the cognition and skills necessary to navigate the system. Specifically, the present study indicated that attitudes such as trust and engagement in the healthcare system and healthcare workers as especially important aspects of health literacy (Gany, Herrera, Avallone, \& Changrani, 2006). Similarly, engagement in healthcare services was another form of attitude that was identified as an aspect of parental health literacy.

The interviews with healthcare providers also explored other individual aspects of health literacy, such as the parents' immigration status. With regards to the immigrant and refugee parents' health literacy level, our results demonstrated the complexity in determining their health literacy. The findings suggested that the parents' native country and previous exposure to Western healthcare systems played a prominent role in influencing their health literacy levels. In addition, parents' culture and shared beliefs regarding health, illness, and intervention were found to play a major role in shaping parental health literacy and their subsequent health behaviors. These findings mirror previous research on asthmatic patients (Poureslami, Rootman, Doyle-Waters, Nimmon, \& FitzGerald, 2011), which found that various aspects of culture informed participants' understanding of the illness and their perception of the culturally competent responses required to meet their health needs. In the present study, the interviews with the physical and mental health providers highlight the importance of acknowledging how cultural beliefs and understanding can continuously shape parents' health literacy and working knowledge regarding their own and their child's illness and well-being. Furthermore, beyond shared cultural understanding, other individual characteristics, such as poverty and traumatic experiences, were also identified as factors influencing health literacy. These findings are consistent with existing research that have demonstrated the influence of socioeconomic status and other demographic factors on health literacy (Yun et al., 2013).

In addition to individual aspects of parental health literacy, our findings suggest that the institutions also play an important role. For many of the immigrant and refugee families, institutions, such as schools, were often the first entry to health services for the children. For example, mandatory mechanisms implemented with schools with regard to health issues such as vaccinations and wellness visits help ensure that many immigrant and refugee children get these health requirements met, as well as enabled for the identification of other health issues. However, mental health issues are often not detected because mandatory mechanisms for mental health screening do not exist. Thus, it is critical for schools to be able to recognize mental health as well as general health problems and illnesses among children, so that they can make appropriate health referrals. While a growing number of studies have focused on using educational settings to stage interventions for various health issues (Bond et al., 2004; Harrell et al., 1996), little attention has been given to mental health literacy interventions. It is likely that existing health and mental healthcare providers can make a major difference by serving as a connector between schools and ethnic-specific communities.

Parental health literacy was also related to health service seeking in a number of ways, such as whether the parents engaged in help-seeking and service utilization behaviors. Parental health literacy influenced the parent's knowledge, attitudes, and beliefs regarding health, the healthcare system as well as health providers. For immigrants and refugees, knowledge regarding existing culturally appropriate resources, logistic difficulties, communication problems, and sustaining patient-provider relationships added unique layers of challenges that had to be navigated in order to access health services for their children. Our findings suggest that it is critical to also assess the provider's own understanding of how the illness and treatment is conceptualized by their immigrant and refugee patients. For some providers, their understanding of core health concepts greatly differed from their patients and were influenced by their own respective cultural understanding of health. These differences could potentially impact the interactions between the service providers and the parents, as well as the parents' help-seeking behaviors on behalf of their children. Healthcare providers with Western conceptualizations of health and literacy need to demonstrate multicultural competency by being cognizant of potential differences in their patients' cultural beliefs and conceptualizations of health and literacy. Healthcare providers also need to be able to accurately assess their own understanding of their immigrant and refugee patients' health-related conceptualizations.

Specific to health-seeking behaviors, our study results indicated that some parents obtained information from ethnic-specific resources first before reaching out to more general resources. Some of these ethnicspecific resources were family and peers, community leaders, ethnicspecific organizations, and cultural brokers such as interpreters and cultural liaisons. Issues such as mistrust, unfamiliarity, and stigma toward the healthcare system were identified as barriers that prevented parents from obtaining services from more general health service providers, such as primary care clinics. However, current studies on health help-seeking and service utilization have mainly focused on the formal sectors of care such as primary care clinics, emergency departments, and other specialty care clinics (Sanders, Federico, Klass, Abrams and Dreyer, 2009; Huang et al., 2006; Abe-Kim et al., 2007). Thus, more research is needed on the relations between these ethnicspecific and informal sources of care, health literacy, and help-seeking.

More distal than health service utilization, our findings suggest that the parental health literacy also impacts their children's health outcomes. The majority of healthcare providers in the study related that the immigrant and refugee parents they served had lower levels of health literacy, especially when using Western conceptualizations of health literacy. The cases cited generally detailed negative health outcomes among the children when their parents had low health literacy. Factors such as cultural differences and the characteristics of the parents were perceived to have some influence on parental health literacy. These factors, when unaccounted for, could contribute to the mixed findings regarding parental health literacy and health outcomes (DeWalt \& Hink, 2009). Another issue highlighted by the providers is that children's health outcomes are often measured using the absence of immediate incidence of illnesses, such as asthmatic attacks or emergency room visits. However, our findings indicated that more proximal outcomes of the child's health were the parent's level of awareness of their limitation regarding their knowledge, their attitudes regarding health and the healthcare system, and their ability to follow up with recommendations. Distal outcomes were found to include both disease amelioration as well as the child's functioning as productive members of society. Regardless of their proximity, both these proximal and distal outcomes can have a major impact on the developmental trajectory of the child.

The results in the present study help provide support for our conceptual model. Parental health literacy was found to impact child's health outcomes by influencing important health behaviors such as help-seeking and decision making. Specifically, high levels of parental literacy enabled parents to make more informed decisions regarding their children's health, including whether to and where to seek care for their children. Parents with high levels of health literacy were thought to better adhere to recommendations and were better able take appropriate actions to contribute to their children's immediate and long-term health outcomes. The findings also supported the addition of important 
variables such as the parent's own trauma and engagement with the healthcare system to the model. These findings are congruent with some of the findings in Wood, Price, Dake, Telljohann, and Khuder (2010) regarding the relations between parental health literacy and children with asthma.

Despite its compelling findings, this study has several limitations. We employed a purposeful sample of healthcare providers in the field. Although there were efforts taken to ensure diversity in the providers' ethnicity, immigrant status, age, years of clinical experience, and population served, the sample consisted of only White and Asian American participants, so there are limits to the scope of the findings. We did not analyze our data based on the unique characteristics of the participants, such as their racial identity and nativity status. It would have been interesting to examine the nuanced in our data with regard to the participants' characteristics. Additionally, this study explored the phenomenon of parental health literacy from the perspectives of healthcare providers rather than parents themselves. The study responses might differ greatly if taken from the perspectives of parents. However, because these clinicians provide care for multiple patients and families, they are in the unique position to inform researchers about their clinical perspectives. The results from this study also suggest that more in-depth studies are needed from the immigrant and refugee parents' perspectives. We specifically recommend that future research examine the rate and prevalence of low health literacy among immigrant and refugee parents using population-based quantitative approaches with a focus on interventions.

\section{Conclusion}

By Western healthcare standards, immigrant and refugee parents were perceived by their healthcare providers as having low levels of health literacy. The parents were perceived by providers as having deficits in various domains of health literacy, such as conceptualization of diseases and well-being, confidence in the healthcare systems, and having the necessary skill base to navigate the healthcare systems. However, the findings indicated that parental health literacy is a multidimensional construct in immigrant and refugee populations. As such, there is a need for healthcare providers and workers servicing such populations to become cognizant of and understand the multidimensionality of health literacy. Healthcare providers should conceptualize health literacy beyond the just spheres of processing and knowledge of health information by also including trust and engagement with the system as important aspects of health literacy. Furthermore, given that these additional aspects were highly contingent on parents' cultural and individual background along with their lived experiences, providers need to take on a more multicultural point of view when working with diverse populations. These expanded notions of health literacy give credence to the expansion of the definition of health literacy itself as the present study demonstrated how parents' understanding and knowledge of health and wellness differ greatly from the conceptualization and treatment by Western healthcare standards. The findings suggest that large systematic changes must be made in the healthcare systems to make them more inclusive of the immigrant and refugee parental health literacy. One such change could be the implementation of more coordinated efforts between the healthcare systems and schools in order to better serve the health literacy needs of the parents.

The results also have implications for current clinical practices. Our findings indicated that screening for the multidimensional construct of parental health literacy during the initial clinical encounter, or even prior to the first visit, is crucial. By assessing the parents' health literacy levels, healthcare providers would be able to tailor their communication and treatment approach to match and potentially improve their patients' health literacy. Our research indicated that trust and cultural competence were major issues in providing effective care for immigrant and refugee parents and their children. Practitioners should do more than examine the patients' conceptualization and ways of communicating and also be able to be mindful of how their own ways of thinking and behaviors might hinder or be helpful during these clinical encounters. Through increasing awareness of their own bias and socialized professional training, providers could better serve and meet their patients' needs. This may help develop the multicultural competency needed to work with immigrant and refugee populations. In sum, practitioners ought to screen for patients' comprehension as well as their own perception of how patients understand health concepts and information, and this needs to be done throughout the clinical relationship. Our results indicate that health literacy is a process that needs to be built from the first clinical encounter and every subsequent encounter as the rapport between provider and patient is being fostered.

In conclusion, the health literacy among immigrants and refugees does have an impact on their help-seeking, service utilization, and consequently their child's health outcomes. Help-seeking in this particular population does not necessarily result in using formal health services. Instead, parents may seek help from informal sources, such as friends, community leaders, and ethnic-specific organizations for health information. As a result, the current service utilization research needs to take informal sources of help-seeking into account. Thus, the current study indicates a need for more research to further explore the issues regarding immigrant and refugee parent's health literacy with the goal of alleviating the low level of health literacy among the marginalized population.

\section{Declaration of conflicting interests}

The authors declared no potential conflicts of interest with respect to the research, authorship, and/or publication of this article.

\section{Funding}

The authors disclosed receipt of the following financial support for the research, authorship, and or publication of this article: This research project is funded by the Minnesota Agricultural Experiment Station (MIN-55-017).

\section{Acknowledgments}

We thank our community health providers, collaborators, and research assistants for their invaluable contributions to the study.

\section{Appendix A. Health and Mental Health Literacy Questionnaire}

1. As you know, we are researching health literacy among immigrants and refugees. When you hear the phrase health literacy (mental health literacy), how do you understand it? How would you define health literacy (mental health literacy)?

2. How does your clients' health literacy (mental health literacy) impact their ability to understand health or mental health communication? (For example, recommendations or instructions.)

3. How do you think that your clients' health literacy (mental health literacy) influences their health or mental health outcomes?

4. What role do you think health literacy (mental health literacy) has on health disparity for the immigrants and refugees you work with?

5. How do you see the health literacy (mental health literacy) of immigrant/refugee parents impacting their children's health or mental health outcomes?

6. When an immigrant/refugee child has a health or mental health problem, how does the parents' health literacy impact help-seeking behavior?

7. If the children in the immigrant/refugee communities are struggling with health or mental health problems, where or whom do the parents ask for help? 


\section{References}

Abe-Kim, J., Takeuchi, D. T., Hong, S., Zane, N., Sue, S., Spencer, M. S., ... Alegria, M. (2007) Use of mental health-related services among immigrant and US-born Asian Americans: Results from the national Latino and Asian American study. American Journal of Public Health, 97(1), 91-98 (doi: AJPH.2006.098541 [pii]).

Ali, N. K. (2012). Are we training residents to communicate with low health literacy patients? Journal of Community Hospital Internal Medicine Perspectives, 2(4).

Baker, D. W. (2006). The meaning and the measure of health literacy. Journal of General Internal Medicine, 21(8), 878-883.

Bennett, I. M., Chen, J., Soroui, J. S., \& White, S. (2009). The contribution of health literacy to disparities in self-rated health status and preventive health behaviors in olde adults. Annals of Family Medicine, 7(3), 204-211. http://dx.doi.org/10.1370/afm.940.

Berkman, N. D., Sheridan, S. L., Donahue, K. E., Halpern, D. J., \& Crotty, K. (2011). Low health literacy and health outcomes: An updated systematic review. Annals of Internal Medicine, 155(2), 97-107.

Bond, L., Patton, G., Glover, S., Carlin, J. B., Butler, H., Thomas, L., \& Bowes, G. (2004). The gatehouse project: Can a multilevel school intervention affect emotional wellbeing and health risk behaviours? Journal of Epidemiology and Community Health, 58(12), 997-1003 (doi:58/12/997 [pii]).

Braun, V., \& Clarke, V. (2006). Using thematic analysis in psychology. Qualitative Research in Psychology, 3(2), 77-101.

Coffman, M. J., \& Norton, C. K. (2010). Demands of immigration, health literacy, and depression in recent Latino immigrants. Home Healthcare Management \& Practice, 22(2), 116-122.

Dennis, S., Williams, A., Taggart, J., Newall, A., Denney-Wilson, E., Zwar, N., ... Harris, M. F. (2012). Which providers can bridge the health literacy gap in lifestyle risk factor modification education: A systematic review and narrative synthesis. BMC Family Practice, 13(no. 1), 44.

DeWalt, D. A., \& Hink, A. (2009). Health literacy and child health outcomes: A systematic review of the literature. Pediatrics, 124(Suppl. 3), S265-S274. http://dx.doi.org/10. 1542/peds.2009-1162B.

Gany, F. M., Herrera, A. P., Avallone, M., \& Changrani, J. (2006). Attitudes, knowledge, and health-seeking behaviors of five immigrant minority communities in the prevention and screening of cancer: A focus group approach. Ethnicity and Health, 11(1), 19-39.

Glascoe, F. P., \& Dworkin, P. H. (1995). The role of parents in the detection of developmental and behavioral problems. Pediatrics, 95(6), 829-836.

Han, H. R., Kim, J., Kim, M. T., \& Kim, K. B. (2011). Measuring health literacy among immigrants with a phonetic primary language: A case of Korean American women. Journal of Immigrant and Minority Health, 13(2), 253-259.

Harrell, J. S., McMurray, R. G., Bangdiwala, S. I., Frauman, A. C., Gansky, S. A., \& Bradley, C. B. (1996). Effects of a school-based intervention to reduce cardiovascular disease risk factors in elementary-school children: The cardiovascular health in children (CHIC) study. The Journal of Pediatrics, 128(6), 797-805.

Harrington, K. F., Haven, K. M., Bailey, W. C., \& Gerald, L. B. (2013). Provider perceptions of parent health literacy and effect on asthma treatment recommendations and instructions. Pediatric Allergy, Immunology, and Pulmonology, 26(2), 69-75.

Hironaka, L. K., \& Paasche-Orlow, M. K. (2008). The implications of health literacy on patient-provider communication. Archives of Disease in Childhood, 93(5), 428-432.

Huang, Z. J., Stella, M. Y., \& Ledsky, R. (2006). Health status and health service access and use among children in US immigrant families. Journal Information, 96(4).

Jorm, A. F. (2012). Mental health literacy: Empowering the community to take action for better mental health. American Psychologist, 67(3), 231.

Kreps, G. L., \& Sparks, L. (2008). Meeting the health literacy needs of immigrant populations. Patient Education and Counseling, 71(3), 328-332.

Kripalani, S., Henderson, L. E., Chiu, E. Y., Robertson, R., Kolm, P., \& Jacobson, T. A. (2006) Predictors of medication self-management skill in a low-literacy population. Journal of General Internal Medicine, 21(8), 852-856.

Nielsen-Bohlman, L., Panzer, A., \& Kindig, D. (2004). Committee on health literacy. Health literacy: A prescription to end confusion.
Pew Research Center (2012). The rise of the Asian Americans. (Retrieved from:) http:// www.pewsocialtrends.org/2012/06/19/the-rise-of-asian-americans/

Poureslami, I., Rootman, I., Doyle-Waters, M. M., Nimmon, L., \& FitzGerald, J. M. (2011). Health literacy, language, and ethnicity-related factors in newcomer asthma patients to Canada: A qualitative study. Journal of Immigrant and Minority Health, 13(2), 315-322.

Ratzan, S. C., \& Parker, R. M. (2000). Introduction. In C. R. Selden, M. Zorn, S. C. Ratzan, \& R. M. Parker (Eds.), National library of medicine current bibliographies in medicine: Health literacy. NLM pub. no. CBM 2000-1. Bethesda, MD: National Institutes of Health, U.S. Department of Health and Human Services.

Rosenthal, M. S., Socolar, R. R., DeWalt, D. A., Pignone, M., Garrett, J., \& Margolis, P. A. (2007). Parents with low literacy report higher quality of parent-provider relationships in a residency clinic. Ambulatory Pediatrics, 7(1), 51-55.

Sanders, L. M., Thompson, V. T., \& Wilkinson, J. D. (2007). Caregiver health literacy and the use of child health services. Pediatrics, 119(1), e86-e92 (doi:119/1/e86 [pii]).

Sanders, L. M., Federico, S., Klass, P., Abrams, M. A., \& Dreyer, B. (2009a). Literacy and child health: A systematic review. Archives of Pediatrics \& Adolescent Medicine, 163(2), 131-140.

Sanders, L. M., Shaw, J. S., Guez, G., Baur, C., \& Rudd, R. (2009b). Health literacy and child health promotion: Implications for research, clinical care, and public policy. Pediatrics, 124(Suppl. 3), S306-S314. http://dx.doi.org/10.1542/peds.2009-1162G.

Sørensen, K., Van den Broucke, S., Fullam, J., Doyle, G., Pelikan, J., Slonska, Z., \& Brand, H. (2012). Health literacy and public health: A systematic review and integration of definitions and models. BMC Public Health, 12(1), 1

Tsai, T. I., \& Lee, S. Y. D. (2015). Health literacy as the missing link in the provision of immigrant healthcare: A qualitative study of southeast Asian immigrant women in Taiwan. (International journal of nursing studies).

Turner, T., Cull, W. L., Bayldon, B., Klass, P., Sanders, L. M., Frintner, M. P., ... Dreyer, B. (2009). Pediatricians and health literacy: Descriptive results from a national survey. Pediatrics, 124(Suppl. 3), S299-S305. http://dx.doi.org/10.1542/peds.2009-1162F.

Wood, M. R., Price, J. H., Dake, J. A., Telljohann, S. K., \& Khuder, S. A. (2010). African American parents'/guardians' health literacy and self-efficacy and their child's level of asthma control. Journal of Pediatric Nursing, 25(5), 418-427.

World Health Organization (1998). The world health report 1998: Life in the 21st century a vision for all. World Health Organization.

Yin, H. S., Johnson, M., Mendelsohn, A. L., Abrams, M. A., Sanders, L. M., \& Dreyer, B. P. (2009). The health literacy of parents in the United States: A nationally representative study. Pediatrics, 124(Suppl. 3), S289-S298. http://dx.doi.org/10.1542/peds. 2009-1162E.

Yun, K., Fuentes-Afflick, E., Curry, L. A., Krumholz, H. M., \& Desai, M. M. (2013). Parental immigration status is associated with Children's healthcare utilization: Findings from the 2003 new immigrant survey of US legal permanent residents. Maternal and Child Health Journal, 17(10), 1913-1921.

Belle P. Khuu is a doctoral student at the University of Minnesota School of Social Work, St. Paul, MN, USA

Hee Y. Lee is a professor at the University of Minnesota School of Social Work, St. Paul, MN, USA.

Anne Q.Zhou is a doctoral student at the University of Minnesota Department of Psychology, Minneapolis, MN, USA.

Jihee Shin is a graduate student at the University of Minnesota School of Social Work, St. Paul, MN, USA.

Richard M. Lee is a professor University of Minnesota Department of Psychology, Minneapolis, MN, USA. 